\title{
Combined Heat and Power Dynamic Economic Dispatch with Emission Limitations Using Hybrid DE-SQP Method
}

\author{
A. M. Elaiw, ${ }^{1,2}$ X. Xia, ${ }^{3}$ and A. M. Shehata ${ }^{2}$ \\ ${ }^{1}$ Department of Mathematics, Faculty of Science, King Abdulaziz University, P.O. Box 80203, Jeddah 21589, Saudi Arabia \\ ${ }^{2}$ Department of Mathematics, Faculty of Science, Al-Azhar University, Assiut 71511, Egypt \\ ${ }^{3}$ Centre of New Energy Systems, Department of Electrical, Electronic and Computer Engineering, University of Pretoria, \\ Pretoria 0002, South Africa
}

Correspondence should be addressed to A. M. Elaiw; a_m_elaiw@yahoo.com

Received 28 August 2013; Accepted 1 October 2013

Academic Editor: Jinde Cao

Copyright (C) 2013 A. M. Elaiw et al. This is an open access article distributed under the Creative Commons Attribution License, which permits unrestricted use, distribution, and reproduction in any medium, provided the original work is properly cited.

Combined heat and power dynamic economic emission dispatch (CHPDEED) problem is a complicated nonlinear constrained multiobjective optimization problem with nonconvex characteristics. CHPDEED determines the optimal heat and power schedule of committed generating units by minimizing both fuel cost and emission simultaneously under ramp rate constraints and other constraints. This paper proposes hybrid differential evolution (DE) and sequential quadratic programming (SQP) to solve the CHPDEED problem with nonsmooth and nonconvex cost function due to valve point effects. DE is used as a global optimizer, and SQP is used as a fine tuning to determine the optimal solution at the final. The proposed hybrid DE-SQP method has been tested and compared to demonstrate its effectiveness.

\section{Introduction}

Recently, combined heat and power (CHP) units, known as cogeneration or distributed generation, have played an increasingly important role in the utility industry. CHP units can provide not only electrical power but also heat to the customers. While the efficiency of the normal power generation is between $50 \%$ and $60 \%$, the power and heat cogeneration increases the efficiency to around 90\% [1]. Besides thier high efficiency, CHP units reduce the emission of gaseous pollutants $\left(\mathrm{SO}_{2}, \mathrm{NO}_{x}, \mathrm{CO}\right.$, and $)$ by about $13-18 \%$ [2].

In order to utilize the integrated CHP system more $\mathrm{CO}_{2}$ economically, combined heat and power economic dispatch (CHPED) problem is applied. The objective of the CHPED problem is to determine both power generation and heat production from units by minimizing the fuel cost such that both heat and power demands are met, while the combined heat and power units are operated in a bounded heat versus power plane. For most CHP units the heat production capacities depend on the power generation. This mutual dependency of the CHP units introduces a complication to the problem [3]. In addition, considering valve point effects in the CHPED problem makes the problem nonsmooth with multiple local optimal point which makes finding the global optimal challenging.

In the literature, several optimization techniques have been used to solve the CHPED problem with complex objective functions or constraints such as Lagrangian relaxation (LR) $[4,5]$, semidefinite programming (SDP) [6], augmented Lagrange combined with Hopfield neural network [7], harmony search (HS) algorithm $[1,8]$, genetic algorithm (GA) [9], ant colony search algorithm (ACSA) [10], mesh adaptive direct search (MADS) algorithm [11], self adaptive real-coded genetic algorithm (SARGA) [3], particle swarm optimization (PSO) $[2,12]$, artificial immune system (AIS) [13], bee colony optimization (BCO) [14], differential evolution [15], and evolutionary programming (EP) [16]. In [2, 13-15], the valve point effects and the transmission line losses are incorporated into the CHPED problem.

In the CHPED formulation the ramp rate limits of the units are neglected. Plant operators, to avoid life-shortening of the turbines and boilers, try to keep thermal stress on the equipments within the safe limits. This mechanical 
constraint is usually transformed into a limit on the rate of change of the electrical output of generators. Such ramp rate constraints link the generator operation in two consecutive time intervals. Combined heat and power dynamic economic dispatch (CHPDED) problem is an extension of CHPED problem where the ramp rate constraint is considered. The primary objective of the CHPDED problem is to determine the heat and power schedule of the committed units so as to meet the predicted heat and electricity load demands over a time horizon at minimum operating cost under ramp rate constraints and other constraints [17]. Since the ramp rate constraints couple the time intervals, the CHPDED problem is a difficult optimization problem. If the ramp rate constraints are not included in the optimization problem, the CHPDED problem is reduced to a set of uncoupled CHPED problems that can easily be solved. In the literature an overwhelming number of reported works deal with CHPED problem; however, the CHPDED problem has only been considered in [17].

The traditional dynamic economic dispatch (DED) problem which considers only thermal units that provide only electric power has been studied by several authors (see the review paper [18]). The emission has been taken into the traditional (DED) formulation in three main approaches. The first approach is to minimize the fuel cost and treat the emission as a constraint with a permissible limit (see, e.g., [19-21]). This formulation, however, has a severe difficulty in getting the trade-off relations between cost and emission [22]. The second approach handles both fuel cost and emission simultaneously as competing objectives [23-25]. The third approach treats the emission as another objective in addition to fuel cost objective. However, the multiobjective optimization problem is converted to a single-objective optimization problem by linear combination of both objectives $[19,26-30]$. In the second and third approaches, the dynamic dispatch problem is referred to as dynamic economic emission dispatch (DEED) which is a multiobjective optimization problem, which minimizes both fuel cost and emission simultaneously under ramp rate constraint and other constraints $[19,24]$. In this paper, we incoroporate the CHP units into the DEED problem. Combined heat and power dynamic economic emission dispatch (CHPDEED) is formulated with the objective to determine the unit power and heat production so that the system's production cost and emission are simultaneously minimized, while the power and heat demands and other constraints are met [17]. The emission has been taken into consideration in the CHPED and CHPDED in [17, 31], respectively. In [17], both fuel cost and emission are simultaneously handled as competing objectives and the multiobjective problem is solved using an enhanced firefly algorithm (FA). In the present paper, the multiobjective optimization problem is converted into a single-objective optimization using the weighting method. This approach yields meaningful result to the decision maker when solved many times for different values of the weighting factor. In [17], the simulation results for test system are shown, but the data of the heat demand is not explicitly tabulated; instead it is expressed graphically (see Figure 12 in [17]). In this case a comparison of our proposed method and FA cannot be performed. In our paper, all the data and the solutions of the test system are available for comparison.

Differential evolution algorithm (DE), which was proposed by Storn and Price [32] is a population based stochastic parallel search technique. DE uses a rather greedy and less stochastic approach to problem solving compared to other evolutionary algorithms. DE has the ability to handle optimization problems with nonsmooth/nonconvex objective functions [32]. Moreover, it has a simple structure and a good convergence property, and it requires a few robust control parameters [32]. DE has been applied to the CHPED and CHPDED problems with non-smooth and non-convex cost functions in [15, 33], respectively.

The DE shares many similarities with evolutionary computation techniques such as genetic algorithms (GA) techniques. The system is initialized with a population of random solutions and searches for optima by updating generations. DE has evolution operators such as crossover and mutation. Although DE seem to be good methods to solve the CHPDEED problem with non-smooth and non-convex cost functions, solutions obtained are just near global optimum with long computation time. Therefore, hybrid methods such as DE-SQP can be effective in solving the CHPDEED problems with valve point effects.

The main contributions of the paper are as follows. (1) A multi-objective optimization problem is formulated using CHPDEED approach. The multi-objective optimization problem is converted into a single-objective optimization using the weighting method. (2) Hybrid DE-SQP method is proposed and validated for solving the CHPDEED problem with nonsmooth and nonconvex objective function. $\mathrm{DE}$ is used as a base level search for global exploration and SQP is used as a local search to fine-tune the solution obtained from DE. (3) The effectiveness of the proposed method is shown for test systems.

\section{Problem Formulation}

In this section we formulate the CHPDEED problem. The system under consideration has three types of generating units, conventional thermal units (TU), CHP units, and heat-only units $(\mathrm{H})$. The power is generated by conventional thermal units and CHP units, while the heat is generated by CHP units and heat-only units. The objective of the CHPDEED problem is to simultaneously minimize the system's production cost and emission so as to meet the predicted heat and power load demands over a time horizon under ramp rate and other constraints. The following objectives and constraints are taken into account in the formulation of the CHPDEED problem.

2.1. Objective Functions. In this section, we introduce the cost and emission functions of three types of generating units, conventional thermal units which produce power only, CHP units which produce both heat and power, and heat-only units which produce heat only. 


\subsubsection{Conventional Thermal Units}

Cost. The cost function curve of a conventional thermal unit can be approximated by a quadratic function [35]. Power plants commonly have multiple valves which are used to control the power output of the unit. When steam admission valves in conventional thermal units are first open, a sudden increase in losses is registered which results in ripples in the cost function $[18,36]$. This phenomenon is called as valve-point effects. The generator with valve-point effects has very different input-output curve compared with smooth cost function. Taking the valve-point effects into consideration, the fuel cost is expressed as the sum of a quadratic and sinusoidal functions $[17,24,25,37]$. Therefore, the fuel cost function of the conventional thermal units is given by

$$
\begin{aligned}
C_{i}^{\mathrm{TU}}\left(P_{i, t}^{\mathrm{TU}}\right)= & a_{i}+b_{i} P_{i, t}^{\mathrm{TU}}+c_{i}\left(P_{i, t}^{\mathrm{TU}}\right)^{2} \\
& +\left|e_{i} \sin \left(f_{i}\left(P_{i, \mathrm{~min}}^{\mathrm{TU}}-P_{i, t}^{\mathrm{TU}}\right)\right)\right|,
\end{aligned}
$$

where $a_{i}, b_{i}$, and $c_{i}$ are positive constants, $e_{i}$ and $f_{i}$ are the coefficients of conventional thermal unit $i$ reflecting valvepoint effects, $P_{i, t}^{\mathrm{TU}}$ is the power generation of conventional thermal unit $i$ during the $t$ th time interval $[t-1, t), P_{i, \mathrm{Tin}}^{\mathrm{TU}}$ is the minimum capacity of conventional thermal unit $i$, and $C_{i}^{\mathrm{TU}}\left(P_{i, t}^{\mathrm{TU}}\right)$ is the fuel cost of conventional thermal unit $i$ to produce $P_{i, t}^{\mathrm{TU}}$.

Emission. The amount of emission of gaseous pollutants from conventional thermal units can be expressed as a combination of quadratic function and exponential function of the unit's active power output [21]. The emission function is given by

$$
E_{i}^{\mathrm{TU}}\left(P_{i, t}^{\mathrm{TU}}\right)=\alpha_{i}+\beta_{i} P_{i, t}^{\mathrm{TU}}+\gamma_{i}\left(P_{i, t}^{\mathrm{TU}}\right)^{2}+\eta_{i} \exp \left(\delta_{i} P_{i, t}^{\mathrm{TU}}\right)
$$

where $E_{i}^{\mathrm{TU}}\left(P_{i, t}^{\mathrm{TU}}\right)$ is the amount of emission from unit $i$ from producing power $P_{i, t}^{\mathrm{TU}}$. Constants $\alpha_{i}, \beta_{i}, \gamma_{i}, \eta_{i}$, and $\delta_{i}$ are the coefficients of the $i$ th unit emission characteristics [24].

\subsubsection{CHP Units}

Cost. A CHP unit has a convex cost function in both power and heat. The form of the fuel cost function of CHP units can be given by $[6,17]$ the following:

$$
\begin{aligned}
C_{j}^{\mathrm{CHP}}\left(P_{j, t}^{\mathrm{CHP}}, H_{j, t}^{\mathrm{CHP}}\right)= & \bar{a}_{j}+\bar{b}_{j} P_{j, t}^{\mathrm{CHP}}+\bar{c}_{j}\left(P_{j, t}^{\mathrm{CHP}}\right)^{2}+\bar{d}_{j} H_{j, t}^{\mathrm{CHP}} \\
& +\bar{e}_{j}\left(H_{j, t}^{\mathrm{CHP}}\right)^{2}+\bar{f}_{j} P_{j, t}^{\mathrm{CHP}} H_{j, t}^{\mathrm{CHP}}
\end{aligned}
$$

where $C_{j}^{\mathrm{CHP}}\left(P_{j, t}^{\mathrm{CHP}}, H_{j, t}^{\mathrm{CHP}}\right)$ is the generation fuel cost of $\mathrm{CHP}$ unit $i$ to produce power $P_{j, t}^{\mathrm{CHP}}$ and heat $H_{j, t}^{\mathrm{CHP}}$. Constants $\bar{a}_{j}, \bar{b}_{j}, \bar{c}_{j}, \bar{d}_{j}, \bar{e}_{j}$, and $\bar{f}_{j}$ are the fuel cost coefficients of CHP unit $j$.
Emission. The emission of gaseous pollutants from CHP units is proportional to their active power output $[17,31]$ :

$$
E_{j}^{\mathrm{CHP}}\left(P_{j, t}^{\mathrm{CHP}}\right)=\left(\bar{\alpha}_{j}+\bar{\beta}_{j}\right) P_{j, t}^{\mathrm{CHP}},
$$

where $\bar{\alpha}_{j}$ and $\bar{\beta}_{j}$ are the emission coefficients of CHP unit $j$.

\subsubsection{Heat-Only Units}

Cost. The cost function of heat-only units can take the following form $[6,17]$ :

$$
C_{k}^{H}\left(H_{k, t}^{H}\right)=\widetilde{a}_{k}+\widetilde{b}_{k} H_{k, t}^{H}+\widetilde{c}_{k}\left(H_{k, t}^{H}\right)^{2},
$$

where $\tilde{a}_{k}, \widetilde{b}_{k}$, and $\widetilde{c}_{k}$ are the fuel cost coefficients of heat-only unit $k$ and they are constants.

Emission. The emission of gaseous pollutants from CHP units is proportional to their heat output $[17,31]$ :

$$
E_{k}^{H}\left(H_{t}^{H}\right)=\left(\widetilde{\alpha}_{k}+\widetilde{\beta}_{k}\right) H_{k, t}^{H},
$$

where $\widetilde{\alpha}_{k}$ and $\widetilde{\beta}_{k}$ are the emission coefficients of heat-only unit $k$.

Let $N$ be the number of dispatch intervals and $N_{p}+N_{c}+$ $N_{h}$ the number of committed units, where $N_{p}$ is the number of conventional thermal units, $N_{c}$ is the number of the CHP units, and $N_{h}$ is the number of the heat-only units. Then the total fuel cost and amount of emission over the dispatch period $[0, N]$ are given, respectively, by

$$
\begin{aligned}
& C(\mathbf{P H})=\sum_{t=1}^{N}\left(\sum_{i=1}^{N_{p}} C_{i}^{\mathrm{TU}}\left(P_{i, t}^{\mathrm{TU}}\right)+\sum_{j=1}^{N_{c}} C_{j}^{\mathrm{CHP}}\left(P_{j, t}^{\mathrm{CHP}}, H_{j, t}^{\mathrm{CHP}}\right)\right. \\
& \left.+\sum_{k=1}^{N_{h}} C_{k}^{H}\left(H_{k, t}^{H}\right)\right) \\
& E(\mathbf{P H})=\sum_{t=1}^{N}\left(\sum_{i=1}^{N_{p}} E_{i}^{\mathrm{TU}}\left(P_{i, t}^{\mathrm{TU}}\right)+\sum_{j=1}^{N_{c}} E_{j}^{\mathrm{CHP}}\left(P_{j, t}^{\mathrm{CHP}}\right)\right. \\
& \left.+\sum_{k=1}^{N_{h}} E_{k}^{H}\left(H_{k, t}^{H}\right)\right)
\end{aligned}
$$

where $\mathbf{P H}=\left(\mathbf{P H}_{1}, \mathbf{P H}_{2}, \ldots, \mathbf{P H}_{t}, \ldots, \mathbf{P H}_{N}\right)^{\prime}, \mathbf{P H}_{t}=\left(\mathbf{P}_{t}^{\mathrm{TU}}\right.$, $\left.\mathbf{P}_{t}^{\mathrm{CHP}}, \mathbf{H}_{t}^{\mathrm{CHP}}, \mathbf{H}_{t}^{H}\right)^{\prime}, \mathbf{P}_{t}^{\mathrm{TU}}=\left(P_{1, t}^{\mathrm{TU}}, P_{2, t}^{\mathrm{TU}}, \ldots, P_{N_{p}, t}^{\mathrm{TU}}\right)^{\prime}, \mathbf{P}_{t}^{\mathrm{CHP}}=$ $\left(P_{1, t}^{\mathrm{CHP}}, P_{2, t}^{\mathrm{CHP}}, \ldots, P_{N_{c}, t}^{\mathrm{CHP}}\right)^{\prime}, \mathbf{H}_{t}^{\mathrm{CHP}}=\left(H_{1, t}^{\mathrm{CHP}}, H_{2, t}^{\mathrm{CHP}}, \ldots, H_{N_{c}, t}^{\mathrm{CHP}}\right)^{\prime}$, and $\mathbf{H}_{t}^{H}=\left(H_{1, t}^{H}, H_{2, t}^{H}, \ldots, H_{N_{h}, t}^{H}\right)^{\prime}$.

2.2. Constraints. There are three kinds of constraints considered in the CHPDEED problem, that is, the equilibrium constraints of power and heat production, the capacity limits of each unit, and the ramp rate limits.

(i) Power Production and Demand Balance

$$
\sum_{i=1}^{N_{p}} P_{i, t}^{\mathrm{TU}}+\sum_{j=1}^{N_{c}} P_{j, t}^{\mathrm{CHP}}=P_{D, t}+\operatorname{Loss}_{t}, \quad t=1, \ldots, N
$$


TABLE 1: Hourly generation (MW) schedule obtained from DED using DE-SQP for 10-unit system.

\begin{tabular}{|c|c|c|c|c|c|c|c|c|c|c|c|}
\hline $\mathrm{H}$ & $P_{1}^{\mathrm{TU}}$ & $P_{2}^{\mathrm{TU}}$ & $P_{3}^{\mathrm{TU}}$ & $P_{4}^{\mathrm{TU}}$ & $P_{5}^{\mathrm{TU}}$ & $P_{6}^{\mathrm{TU}}$ & $P_{7}^{\mathrm{TU}}$ & $P_{8}^{\mathrm{TU}}$ & $P_{9}^{\mathrm{TU}}$ & $P_{10}^{\mathrm{TU}}$ & Loss \\
\hline 1 & 150.0000 & 35.0000 & 73.0000 & 70.3333 & 222.9974 & 55.1682 & 99.2918 & 120.00 & 20.0000 & 10.0000 & 19.7912 \\
\hline 2 & 150.0000 & 35.0000 & 85 & 120.3 & 54 & 29 & 129. & & 48.7980 & 10.7150 & 22.4058 \\
\hline & 150.0 & 00 & 85 & 170. & 21 & 0 & 6 & 120. & 53.5 & 50 & 28.4468 \\
\hline & 150.0000 & 135.0000 & 16 & 218.2899 & 223.5 & 160.0 & 47 & 120. & 80. & 42.0 & 35.4415 \\
\hline & 150.0000 & 00 & 14 & 12 & 17 & 0 & 73 & 00 & 00 & 36 & 84 \\
\hline & 150.0000 & 135.0000 & 315.1962 & 299.7412 & 243. & 160.0 & 129. & 120. & 80.0000 & 43. & 48.0136 \\
\hline & 150.0000 & 176.9470 & 340.0 & 300.0 & & 160.0 & & & & & 52.9470 \\
\hline & 178.2448 & 228.3049 & 340. & 300. & 2 & 160. & 129 & 120 & 80. & 54 & 58.4 \\
\hline & 258.2448 & 8.3049 & 340. & 300 & & 16 & & & & & 70.5 \\
\hline 0 & 289.0490 & 4.5331 & 340.0 & 300.0 & 243. & 160. & 130 & 120 & 80. & 55. & 79.5821 \\
\hline 11 & 368.7363 & 397.1230 & 340. & 300. & 243 & 160. & 13 & 120 & 80. & 55 & 87.8595 \\
\hline 12 & & 439.5807 & & & & & & & & & \\
\hline 13 & 342.1737 & 386.2 & 340. & 30 & & 16 & & 12 & & & 84.4 \\
\hline 14 & 262.1737 & 306.2429 & 340. & 300 & 24 & 160 & 13 & 00 & 00 & 53 & 93 \\
\hline 15 & 182.1737 & 226.2 & 340. & 299 & & & & & & & 58.4 \\
\hline 16 & 150.0000 & 146.2429 & 294.7 & 249. & 2 & 160 & $12 S$ & 00 & 00 & 43 & 43.6398 \\
\hline 17 & 150.0000 & 135.0000 & 258.1720 & 249.5279 & & 160.0 & 128.8 & 120.0000 & & 13. & 39.3459 \\
\hline 18 & 150.0000 & 151.6366 & 298.4749 & 299.5 & 243. & 160.0 & 129. & 120.0000 & 80. & 43.6183 & 48.0511 \\
\hline 19 & 227.2425 & 231.6366 & 299.3393 & 300.0000 & & 160.0000 & 130.0000 & 120.0000 & & 43.5728 & 58.7914 \\
\hline 20 & 307.2425 & 311.6366 & 340.0000 & 300.0000 & & 160.0000 & & 120.0000 & & 55.0000 & 74.8793 \\
\hline 21 & 265.4293 & 301.1183 & 340.0 & 300.0000 & 243.0 & 160.0000 & 130.0000 & 120.0000 & 80. & 55.0000 & 70.5476 \\
\hline 22 & 185.4293 & 221.1183 & 263.3759 & 250.0000 & 225.8767 & 160.0000 & 129.8685 & 120.0000 & 80.0000 & 41.1109 & 48.7801 \\
\hline 23 & 150.0000 & 141.1183 & 183.3759 & 200.0000 & 223.4887 & 155.9437 & 128.7427 & 120.0000 & 50.0000 & 11.1109 & 31.7806 \\
\hline 24 & 150.0000 & 135.0000 & 173.1056 & 180.5739 & 173.7249 & 118.1382 & 128.6826 & 120.0000 & 20.0000 & 10.0000 & 25.2260 \\
\hline
\end{tabular}

TABLE 2: Comparison results of 10-thermal-unit system (cost $\left.\times 10^{6} \$\right)$ for the DED problem.

\begin{tabular}{lcccccc}
\hline Method & EP [34] & PSO [34] & AIS [34] & NSGA-II [24] & IBFA [30] & DE-SQP \\
\hline cost $(\$)$ & 2.5854 & 2.5722 & 2.5197 & 2.5168 & 2.4817 & 2.4659 \\
\hline
\end{tabular}

TABLE 3: Data of the CHP units and heat-only unit system.

\begin{tabular}{lccccccccc}
\hline CHP units & $\bar{a}_{j}$ & $\bar{b}_{j}$ & $\bar{c}_{j}$ & $\bar{d}_{j}$ & $\bar{e}_{j}$ & $\bar{f}_{j}$ & $\bar{\alpha}_{j}$ & $\bar{\beta}_{j}$ & $\mathrm{DR}_{j}^{\mathrm{CHP}}=\mathrm{UR}_{j}^{\mathrm{CHP}}$ \\
\hline$j=1$ & 2650 & 14.5 & 0.0345 & 4.2 & 0.030 & 0.031 & 0.00015 & 0.0015 & 70 \\
$j=2$ & 1250 & 36 & 0.0435 & 0.6 & 0.027 & 0.011 & 0.00015 & 0.0015 & 50 \\
\hline \multicolumn{2}{c}{ Heat-only units } & $H_{k, \max }^{H}$ & $H_{k, \min }^{H}$ & $\widetilde{a}_{k}$ & $\widetilde{b}_{k}$ & $\widetilde{c}_{k}$ & $\widetilde{\alpha}_{j}$ & $\widetilde{\beta}_{j}$ \\
\hline \multicolumn{2}{c}{$k=1$} & 2695.2 & 0 & 950 & 2.0109 & 0.038 & 0.0008 & 0.0010 \\
\hline
\end{tabular}

where $P_{D, t}$ and Loss $_{t}$ are the system power demand and transmission line losses at time $t$ (i.e., the $t$ th time interval), respectively. The $\mathrm{B}$-coefficient method is one of the most commonly used by power utility industry to calculate the network losses. In this method the network losses are expressed as a quadratic function of the unit's power outputs that can be approximated in the following:

$$
\operatorname{Loss}_{t}=\sum_{i=1}^{N_{p}+N_{c}} \sum_{j=1}^{N_{p}+N_{c}} \mathbf{P L}_{i, t} B_{i j} \mathbf{P L}_{j, t}, \quad t=1, \ldots, N
$$

where

$$
\mathbf{P L}_{i, t}= \begin{cases}P_{i, t}^{\mathrm{TU}}, & i=1, \ldots, N_{p}, \\ P_{i-N_{p}, t}^{\mathrm{CHP}}, & i=N_{p}+1, \ldots, N_{p}+N_{c},\end{cases}
$$

and $B_{i j}$ is the $i j$ th element of the loss coefficient square matrix of size $N_{p}+N_{c}$.

(ii) Heat Production and Demand Balance

$$
\sum_{j=1}^{N_{c}} H_{j, t}^{\mathrm{CHP}}+\sum_{k=1}^{N_{h}} H_{k, t}^{H}=H_{D, t}, \quad t=1, \ldots, N
$$

where $H_{D, t}$ is the system heat demand at time $t$.

(iii) Capacity Limits of Conventional Thermal Units

$$
P_{i, \min }^{\mathrm{TU}} \leq P_{i, t}^{\mathrm{TU}} \leq P_{i, \max }^{\mathrm{TU}}, \quad i=1, \ldots, N_{p}, t=1, \ldots, N
$$


TABLE 4: Heat load demand of the three-unit system for 24 hours.

\begin{tabular}{lc}
\hline Time (h) & Demand (MWth) \\
\hline 1 & 390 \\
2 & 400 \\
3 & 410 \\
4 & 420 \\
5 & 440 \\
6 & 450 \\
7 & 450 \\
8 & 455 \\
9 & 460 \\
10 & 460 \\
11 & 470 \\
12 & 480 \\
13 & 470 \\
14 & 460 \\
15 & 450 \\
16 & 450 \\
17 & 420 \\
18 & 435 \\
19 & 445 \\
20 & 450 \\
21 & 445 \\
22 & 435 \\
23 & 400 \\
\hline & 400 \\
\hline
\end{tabular}

where $P_{i, \min }^{\mathrm{TU}}$ and $P_{i, \max }^{\mathrm{TU}}$ are the minimum and maximum power capacity of conventional thermal unit $i$, respectively.

\section{(iv) Capacity Limits of CHP Units}

$$
\begin{array}{r}
P_{j, \text { min }}^{\mathrm{CHP}}\left(H_{j, t}^{\mathrm{CHP}}\right) \leq P_{j, t}^{\mathrm{CHP}} \leq P_{j, \max }^{\mathrm{CHP}}\left(H_{j, t}^{\mathrm{CHP}}\right), \\
j=1, \ldots, N_{c}, \quad t=1, \ldots, N, \\
H_{j, \text { min }}^{\mathrm{CHP}}\left(P_{j, t}^{\mathrm{CHP}}\right) \leq H_{j, t}^{\mathrm{CHP}} \leq H_{j, \max }^{\mathrm{CHP}}\left(P_{j, t}^{\mathrm{CHP}}\right), \\
j=1, \ldots, N_{c}, \quad t=1, \ldots, N,
\end{array}
$$

where $P_{j, \min }^{\mathrm{CHP}}\left(H_{j, t}^{\mathrm{CHP}}\right)$ and $P_{i, \text { max }}^{\mathrm{CHP}}\left(H_{j, t}^{\mathrm{CHP}}\right)$ are the minimum and maximum power limit of CHP unit $j$, respectively, and they are functions of generated heat $\left(H_{j, t}^{\mathrm{CHP}}\right) . H_{j \text {,min }}^{\mathrm{CHP}}\left(P_{j, t}^{\mathrm{CHP}}\right)$ and $H_{j, \max }^{\mathrm{CHP}}\left(P_{j, t}^{\mathrm{CHP}}\right)$ are the heat generation limits of CHP unit $j$ which are functions of generated power $\left(P_{j, t}^{\mathrm{CHP}}\right)$.

\section{(v) Capacity Limits of Heat-Only Units}

$$
H_{k, \min }^{H} \leq H_{k, t}^{H} \leq H_{k, \max }^{H}, \quad k=1, \ldots, N_{h}, t=1, \ldots, N
$$

where $H_{k, \text { min }}^{H}$ and $H_{k, \text { max }}^{H}$ are the minimum and maximum heat capacity of heat-only unit $k$, respectively. (vi) Upper/Down Ramp Rate Limits of Conventional Thermal Units

$$
\begin{gathered}
-D R_{i}^{\mathrm{TU}} \leq P_{i, t+1}^{\mathrm{TU}}-P_{i, t}^{\mathrm{TU}} \leq U R_{i}^{\mathrm{TU}} \\
i=1, \ldots, N_{p}, \quad t=1, \ldots, N-1,
\end{gathered}
$$

where $U R_{i}^{\mathrm{TU}}$ and $D R_{i}^{\mathrm{TU}}$ are the maximum ramp up/down rates for conventional thermal unit $i[18]$.

(vii) Upper/Down Ramp Rate Limits of CHP Units

$$
\begin{gathered}
-D R_{j}^{\mathrm{CHP}} \leq P_{j, t+1}^{\mathrm{CHP}}-P_{j, t}^{\mathrm{CHP}} \leq U R_{j}^{\mathrm{CHP}}, \\
j=1, \ldots, N_{c}, \quad t=1, \ldots, N-1,
\end{gathered}
$$

where $U R_{j}^{\mathrm{CHP}}$ and $D R_{j}^{\mathrm{CHP}}$ are the maximum ramp up/down rates for CHP unit $j$ [17].

2.3. The Optimization Problem. Aggregating the objectives and constraints, the CHPDEED problem can be mathematically formulated as a nonlinear constrained multi-objective optimization problem which can be converted into a singleobjective optimization using the weighting method as

$$
\min _{\mathbf{P H}} F(\mathbf{P H})=w C(\mathbf{P H})+(1-w) E(\mathbf{P H}),
$$

subject to constraints $(8)-(16)$,

where $w \in[0,1]$ is a weighting factor. It will be noted that, when $w=1$, problem (17) determines the optimal amount of the generated heat and power by minimizing the fuel cost regardless of emission and the problem will be referred to as combined heat and power dynamic economic dispatch (CHPDED) problem. If $w=0$, then problem (17) determines the optimal amount of the generated power by minimizing the emission regardless of cost and the problem will be referred to as combined heat and power pure dynamic emission dispatch (CHPPDED).

\section{Differential Evolution Method}

DE is a simple yet powerful heuristic method for solving nonlinear, nonconvex, and nonsmooth optimization problems. $\mathrm{DE}$ algorithm is a population based algorithm using three operators; mutation, crossover, and selection to evolve from randomly generated initial population to final individual solution [32]. In the initialization a population of NP target vectors (parents) $X_{i}=\left\{x_{1 i}, x_{2 i}, \ldots, x_{D i}\right\}, i=1,2, \ldots, \mathrm{NP}$, is randomly generated within user-defined bounds, where $D$ is the dimension of the optimization problem. Let $X_{i}^{G}=$ $\left\{x_{1 i}^{G}, x_{2 i}^{G}, \ldots, x_{D i}^{G}\right\}$ be the individual $i$ at the current generation $G$. A mutant vector $V_{i}^{G+1}=\left(v_{1 i}^{G+1}, v_{2 i}^{G+1}, \ldots, v_{D i}^{G+1}\right)$ is generated according to

$$
\begin{gathered}
V_{i}^{G+1}=X_{r_{1}}^{G}+\mathscr{F} \times\left(X_{r_{2}}^{G}-X_{r_{3}}^{G}\right), \\
r_{1} \neq r_{2} \neq r_{3} \neq i, \quad i=1,2, \ldots, \mathrm{NP},
\end{gathered}
$$


TABLE 5: Hourly heat and power schedule obtained from CHPDED.

\begin{tabular}{|c|c|c|c|c|c|c|c|c|c|c|c|c|c|}
\hline$P_{1}^{\mathrm{TU}}$ & $P_{2}^{\mathrm{TU}}$ & $P_{3}^{\mathrm{TU}}$ & $P_{4}^{\mathrm{TU}}$ & $P_{5}^{\mathrm{TU}}$ & $P_{6}^{\mathrm{TU}}$ & $P_{7}^{\mathrm{TU}}$ & $P_{8}^{\mathrm{TU}}$ & $P_{1}^{\mathrm{CHP}}$ & $P_{2}^{\mathrm{CHP}}$ & 3 & $H_{1}^{\mathrm{CHP}}$ & $\mathrm{H}_{2}^{\mathrm{CHP}}$ & $H_{1}^{\mathrm{H}}$ \\
\hline 50.0000 & 35.0000 & 74.5372 & 2.0784 & 4.5129 & 4.4 & .0000 & 10.0000 & 236.8041 & 0.1974 & 0 & 57.3450 & & \\
\hline 50.0000 & 35.0000 & 35 & 84 & & & & & & & & & & \\
\hline 50 & & & & & & & & & & & & & \\
\hline 50.0 & 0 & 06 & & & 12 & & & & & & & & \\
\hline 0 & & & & & & & & & & & & & \\
\hline 150. & 0 & 66 & & & & & & & & & & & \\
\hline 0 & & & & & & & & & & & & & \\
\hline 89. & & 00 & & & & & & & & & & & \\
\hline 265. & & & & & & & & & & & & & \\
\hline 4 & & 00 & & & & & & & & & & & \\
\hline 7 & & & & & & & & & & & & & \\
\hline 2367 & 172 & 00 & & & & & & & & & & & \\
\hline 3 & & 00 & & & & & & & & & & & \\
\hline 4 & & 00 & & & & & & & & & & & \\
\hline 5 & & 00 & & & & & & & & & & & \\
\hline 00 & & 30 & & & & & & & & & & & \\
\hline 00 & & 99 & & & & & & & & & & & \\
\hline 0 & & & & & & & & & & & & & \\
\hline 9229 & & & & & & & & & & & & & \\
\hline 0309 & 6 & & & & & & & & & & & & \\
\hline 1272. & 7 & 340. & & & & & & & & & & & \\
\hline 2192.4577 & 220.8037 & 260.6669 & 00 & & & & & & & & & & 2 \\
\hline 3150.0000 & & & & & & & & & & & & & \\
\hline 4150.0000 & 5.0000 & 00.6669 & 7.0362 & 49 & 162 & 6 & 00 & 572 & 10 & 24 & 6 & 135 & 195.52 \\
\hline
\end{tabular}
Cost $(\$)=2.5257 \times 10^{6}$. Emission $(\mathrm{lb})=2.8287 \times 10^{5}$. Total loss $(\mathrm{MW})=1.3443 \times 10^{3}$.

TABLE 6: Hourly heat and power schedule obtained from CHPDEED $(w=0.5)$.

\begin{tabular}{|c|c|c|c|c|c|c|c|c|c|c|c|c|c|c|}
\hline & $P_{1}^{\mathrm{TU}}$ & $P_{2}^{\mathrm{TU}}$ & $P_{3}^{\mathrm{TU}}$ & $P_{4}^{\mathrm{TU}}$ & $P_{5}^{\mathrm{TU}}$ & $P_{6}^{\mathrm{TU}}$ & $P_{7}^{\mathrm{TU}}$ & $P_{8}^{\mathrm{TU}}$ & $P_{1}^{\mathrm{CHP}}$ & $P_{2}^{\mathrm{CHP}}$ & Loss & $H_{1}^{\mathrm{CHP}}$ & $H_{2}^{\mathrm{CHP}}$ & $H_{1}^{H}$ \\
\hline 1 & 0.0000 & 135.0 & .5875 & .0188 & 2.5 & 90 & 0 & 10.0000 & 238.1 & 4 & 5 & 49.6499 & 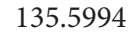 & - \\
\hline & & & & & & & & & & & & & & \\
\hline & 0 & & & & & & & & & & & & & \\
\hline & 0.0000 & 00 & 55 & 3. & 0 & & & & & & & & & \\
\hline & 0 & 0 & 5 & & & & & & & & & & & \\
\hline & 50. & 06 & & & & & & & & & & & & \\
\hline & 5 & & & & & & & & & & & & & \\
\hline & 1 & 23 & 85 & & & & & & & & & & & \\
\hline & 6 & & & & & & & & & & & & & \\
\hline & 6 & 08 & & & & & & & & & & & & \\
\hline & & & & & & & & & & & & & & \\
\hline & & 74 & & & & & & & & & & & & \\
\hline & & & & & & & & & & & & & & \\
\hline & & & & & & & & & & & & & & \\
\hline & & & & & & & & & & & & & & \\
\hline & & 37 & & & & & & & & & & & & \\
\hline & 0.0000 & & & & & & & & & & & & & \\
\hline & & 52 & & & & & & & & & & & & \\
\hline & & & & & & & & & & & & & & \\
\hline & 51 & & 00 & 00 & & & & & & & & & & \\
\hline & & 301. & & & & & & & & & & & & \\
\hline & & 221 & 260 & & & & & & 23 & & & & & \\
\hline & & & & & & & & & & & & & & \\
\hline & 150.0000 & 135.0000 & 0.0000 & 150.0000 & 525 & 125 & 178 & 000 & 235 & 36 & 35 & 91 & 11 & 204.73 \\
\hline
\end{tabular}
Cost $(\$)=2.5295 \times 10^{6}$. Emission $(\mathrm{lb})=2.7209 \times 10^{5}$. Total loss $(\mathrm{MW})=1.3439 \times 10^{3}$. 
TABLE 7: Hourly heat and power schedule obtained from CHPPDED.

\begin{tabular}{|c|c|c|c|c|c|c|c|c|c|c|c|c|c|c|}
\hline $\mathrm{H}$ & $P_{1}^{\mathrm{TU}}$ & $P_{2}^{\mathrm{TU}}$ & $P_{3}^{\mathrm{TU}}$ & $P_{4}^{\mathrm{TU}}$ & $P_{5}^{\mathrm{TU}}$ & $P_{6}^{\mathrm{TU}}$ & $P_{7}^{\mathrm{TU}}$ & $P_{8}^{\mathrm{TU}}$ & $P_{1}^{\mathrm{CHP}}$ & $P_{2}^{\mathrm{CH}}$ & Loss & $H_{1}^{\mathrm{CHP}}$ & $\mathrm{H}_{2}^{\mathrm{CHP}}$ & $H_{1}^{H}$ \\
\hline & 50.0000 & 35.0000 & 3.0000 & 0.0000 & 4340 & 2 & 1020 & 55 & 247 & 125.8 & 21.8228 & 00 & .4722 & 52 \\
\hline & 0.0000 & & 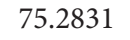 & & & & & r & & & & & & \\
\hline & 150. & 17 & 08.1787 & 8 & 2 & 6 & 00 & 5 & 247 & .8 & b & 0.0 & & 39 \\
\hline & 187.3290 & 229 & 677 & 27 & 00 & 00 & 00 & 55 & 247 & 5.8 & 3 & 0.0 & 74 & 926 \\
\hline 5 & 209.9448 & 210.5929 & 152.0706 & 866 & 00 & 130.0000 & .0000 & 55 & 247 & 125.8 & 42.6 & 0.0 & 974 & 407.5926 \\
\hline & 252.6588 & 252.9491 & 188.3610 & 188.4287 & 000 & 130.0000 & 80.0000 & 55 & 247 & 125.8 & 52.1977 & 0.0 & 244 & 424.4756 \\
\hline & 272.2261 & 272.7171 & 208. & 186 & 00 & 130.0000 & 80.0000 & 5 & 247 & 5.8 & $5 \%$ & 0.0 & 37 & 42 \\
\hline & 290.5 & 33 & 77 & 57 & & 1 & 0 & 5 & 247 & 125.8 & & 0.0 & & \\
\hline & 00 & 32 & 2 & & & & & 55 & & & & & & \\
\hline 10 & 55 & 346 & 3 & 0 & 00 & 00 & 00 & 5 & 247 & 5.8 & 4 & & & 26 \\
\hline 11 & 379.2210 & 85 & 00 & 00 & 00 & 13 & 00 & 5 & 247 & 5.8 & 9 & 0.0 & & 44 \\
\hline 12 & 403.5504 & 403. & 340 & 300 & 00 & 13 & 00 & 55 & 24 & 125.8 & 9 & 0.0 & 345 & 155 \\
\hline 13 & 361.7512 & 362.0812 & 337.4700 & 300.0000 & 000 & 130 & 000 & 55 & 247 & 125.8 & 87 & 0.0 & 189 & 437 \\
\hline 14 & 323.7805 & 324 & 276.7607 & 275. & 160. & 130 & 000 & 55 & 247 & 125.8 & 7 & 0.0 & 363 & 43 \\
\hline 15 & 291 & 292 & & & & & & 55 & & & & & & \\
\hline 16 & 229.7976 & 230 & 167 & & & & & 5 & & & & & & \\
\hline 17 & 99 & 74 & 1 & 1 & & & & 5 & & & & 0 & & 38 \\
\hline 18 & 252.7542 & 253 & 188 & 81 & 00 & 13 & 00 & 55 & 2 & 5.8 & & 0 & 91 & 40 \\
\hline 19 & 288.2429 & 288.7410 & 226.6332 & 237.2113 & 160.0000 & 130.0000 & 80.0000 & 55 & 247 & 125.8 & 62.6285 & 0.0 & 27.4724 & 417.5276 \\
\hline 20 & 335.1319 & 335.4397 & 294.6392 & 287.2113 & 160.0000 & 130 & 80.0000 & 55 & 247 & 125.8 & 22 & 0.0 & 390 & 418.6610 \\
\hline 21 & 332.6192 & 333.0535 & 282.3523 & 252.7233 & 160.0000 & 130.0000 & 80.0000 & 55 & 247 & 125.8 & 74.5483 & 0.0 & 32.3100 & 412.6900 \\
\hline 22 & 252.6192 & 253.0535 & 202.3523 & 202.7233 & 149.4115 & 112.3565 & 80.0000 & 55 & 247 & 125.8 & 52.3163 & 0.0 & 27.3503 & 407.6497 \\
\hline 23 & 172.6192 & 173.0535 & 122.3523 & 152.7233 & 135.8629 & 102.0552 & 80.0000 & 55 & 247 & 125.8 & 34.4664 & 0.0 & 25.1547 & 374.8453 \\
\hline 24 & 50.0000 & 35.0000 & 90.3380 & 102.7233 & 354 & 96 & 00 & 5 & 247 & 1 & 771 & $0 . .0$ & .5331 & 368.4669 \\
\hline
\end{tabular}

Cost $(\$)=2.6945 \times 10^{6}$. Emission $(\mathrm{lb})=2.4195 \times 10^{5}$. Total loss $(\mathrm{MW})=1.3684 \times 10^{3}$.

with randomly chosen integer indexes $r_{1}, r_{2}, r_{3} \in\{1,2$, $\ldots, \mathrm{NP}\}$. Here $\mathscr{F}$ is the mutation factor.

According to the target vector $X_{i}^{G}$ and the mutant vector $V_{i}^{G+1}$, a new trial vector (offspring) $U_{i}^{G+1}=\left\{u_{1 i}^{G+1}\right.$, $\left.u_{2 i}^{G+1}, \ldots, u_{D i}^{G+1}\right\}$ is created with

$$
u_{j i}^{G+1}= \begin{cases}v_{j i}^{G+1}, & \text { if }(\operatorname{rand}(j) \leq \mathrm{CR}) \text { or } j=r n b(i), \\ x_{j i}^{G}, & \text { otherwise, }\end{cases}
$$

where $j=1,2, \ldots, D, i=1,2, \ldots, \mathrm{NP}$ and $\operatorname{rand}(j)$ is the $j$ th evaluation of a uniform random number between $[0,1]$. CR $\epsilon$ $[0,1]$ is the crossover constant which has to be determined by the user. $r n b(i)$ is a randomly chosen index from $1,2, \ldots, D$ which ensures that $U_{i}^{G+1}$ gets at least one parameter from $V_{i}^{G+1}[32]$.

The selection process determines which of the vectors will be chosen for the next generation by implementing one-to-one competition between the offsprings and their corresponding parents. If $f$ denotes the function to be minimized, then

$$
X_{i}^{G+1}= \begin{cases}U_{i}^{G+1} & \text { if } f\left(U_{i}^{G+1}\right) \leq f\left(X_{i}^{G}\right), \\ X_{i}^{G} & \text { otherwise, }\end{cases}
$$

where $i=1,2, \ldots$, NP. The value of $f$ of each trial vector $U_{i}^{G+1}$ is compared with that of its parent target vector $X_{i}^{G}$. The above iteration process of reproduction and selection will continue until a user-specified stopping criteria is met.

In this paper, we define the evaluation function for evaluating the fitness of each individual in the population in $\mathrm{DE}$ algorithm as follows:

$$
\begin{aligned}
f= & F+\lambda_{1} \sum_{t=1}^{N}\left(\sum_{i=1}^{N_{p}} P_{i, t}^{\mathrm{TU}}+\sum_{j=1}^{N_{c}} P_{j, t}^{\mathrm{CHP}}-\left(P_{D, t}+\text { Loss }_{t}\right)\right)^{2} \\
& +\lambda_{2} \sum_{t=1}^{N}\left(\sum_{j=1}^{N_{c}} H_{j, t}^{\mathrm{CHP}}+\sum_{k=1}^{N_{h}} H_{k, t}^{H}-H_{D, t}\right)^{2}
\end{aligned}
$$

where $\lambda_{1}$ and $\lambda_{2}$ are penalty values. Then the objective is to find $f_{\text {min }}$, the minimum evaluation value of all the individuals in all iterations. The penalty term reflects the violation of the equality constraints. Once the minimum of $f$ is reached, the equality constraints are satisfied.

\section{Sequential Quadratic Programming Method}

SQP method can be considered as one of the best nonlinear programming methods for constrained optimization problems [38]. It outperforms every other nonlinear programming method in terms of efficiency, accuracy, and percentage of successful solutions over a large number of test problems. The method closely resembles Newton's method for 


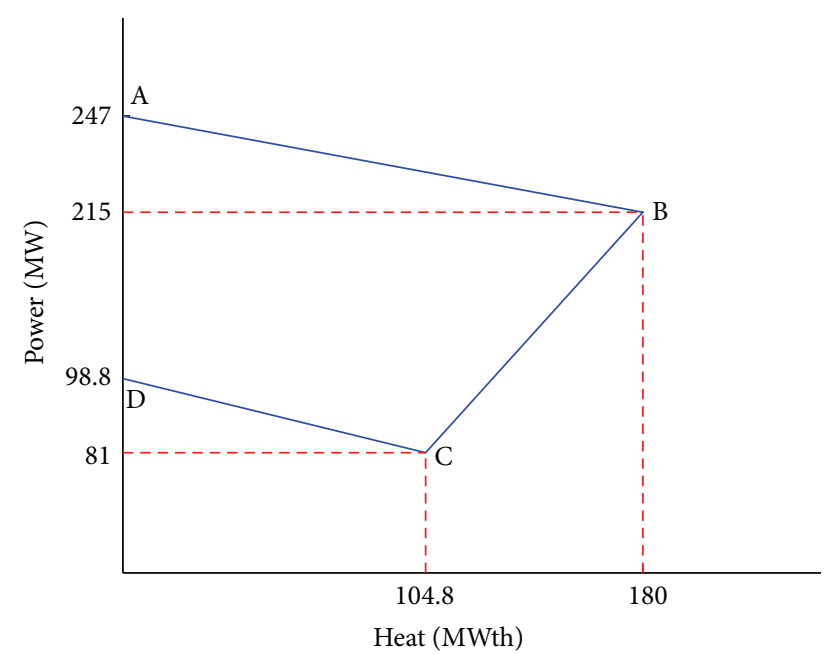

FIgURE 1: Heat-power feasible operating region for CHP unit 1.

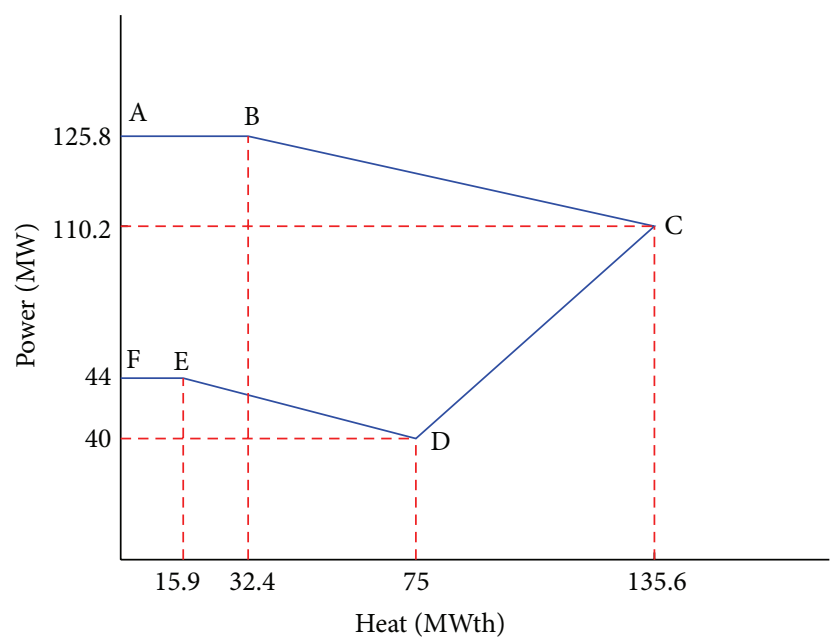

FIGURE 2: Heat-power feasible operating region for CHP unit 2.

constrained optimization, just as is done for unconstrained optimization. At each iteration, an approximation is made of the Hessian of the Lagrangian function using BroydenFletcher-Goldfarb-Shanno (BFGS) quasi-Newton updating method. The result of the approximation is then used to generate a quadratic programming (QP) subproblem whose solution is used to form a search direction for a line search procedure. Since the objective function of the CHPDEED problem is non-convex and non-smooth, SQP ensures a local minimum for an initial solution. In this paper, DE is used as a global search and finally the best solution obtained from DE is given as initial condition for SQP method as a local search to fine-tune the solution. SQP simulations can be computed by the fmincon code of the MATLAB Optimization Toolbox.

\section{Simulation Results}

In this section we present two examples. The first example shows the efficiency of the proposed DE-SQP method for the DED problem. In the second example, the hybrid DE-SQP method is applied to the CHPDEED problem. In DE-SQP method, the control parameters are chosen as NP $=80, \mathscr{F}=$ 0.423 and $\mathrm{CR}=0.885$. The maximum number of iterations are selected as 20,000 . The results represent the average of 30 runs of the proposed method. All computations are carried out by MATLAB program.

Example 1. This example consists of ten conventional thermal units to investigate the effectiveness of the proposed DESQP technique in solving the DED problem with valve point effects and transmission line losses. The technical data of the units as well as the demand for the 10-unit system are taken from [24]. The best solution of the DED problem is given in Table 1. Comparison between our proposed method (DESQP) and other methods is given in Table 2. It is observed that the proposed method reduces the total generation cost better than the other methods reported in the literature.

Example 2. This example is 11-unit system (eight conventional thermal units, two CHP units, and one heat-only unit) for solving the CHPDED, CHPDEED, and CHPPDED problems using DE-SQP method. We shall solve the CHPDEED problem when $w=0.5$, in addition to the CHPDED and CHPPDED problems which correspond to $w=1$ and $w=0$, respectively. The technical data of conventional thermal units, the matrix $B$, and the demand are taken from the 10 -unit system presented in [24]. The 5th and 8th conventional units in [24] were replaced by two CHP units. The technical data of the two CHP units and the heat-only unit are taken from [17] and are given in Table 3. The heat demand for 24 hours is given in Table 4. The feasible operating regions of the two CHP units are given in Figures 1 and 2 (see $[4,14]$ ).

The best solutions of the CHPDED, CHPDEED, and CHPPDED problems for DE-SQP algorithm are given in Tables 5, 6, and 7, respectively. The best cost, the amount of emission, and the transmission line losses are also given in Tables 5-7. It is seen that the cost is $2.5257 \times 10^{6} \$$ under CHPDED, but it increases to $2.6945 \times 10^{6} \$$ under CHPPDED. The emission obtained from CHPDED is $2.8287 \times 10^{5} \mathrm{lb}$, but it decreases to $2.4195 \times 10^{5} \mathrm{lb}$ under CHPPDED. Under the CHPDEED problem, the cost is $2.5295 \times 10^{6} \$$ which is more than $2.5257 \times 10^{6} \$$ and less than $2.6945 \times 10^{6} \$$. Moreover, the emission is $2.7209 \times 10^{5} \mathrm{lb}$ which is less than $2.8287 \times 10^{5} \mathrm{lb}$ and more than $2.4195 \times 10^{5} \mathrm{lb}$.

\section{Conclusion}

This paper presents a hybrid method combining differential evolution (DE) and sequential quadratic programming (SQP) for solving dynamic dispatch (CHPDED, CHPDEED, and CHPPDED) problems with valve-point effects including generator ramp rate limits. In this paper, DE is first applied to find the best solution. This best solution is given to SQP as an initial condition that fine tunes the optimal solution at the final. The feasibility and efficiency of the DE-SQP were illustrated by conducting case studies with system consisting of eight conventional thermal units, two CHP units, and one heat-only unit. 


\section{Conflict of Interests}

The authors declare that there is no conflict of interests regarding the publication of this paper.

\section{Acknowledgment}

This work was funded by the Deanship of Scientific Research (DSR), King Abdulaziz University, Jeddah, under Grant no. (130-107-D1434). The authors, therefore, acknowledge with thanks DSR technical and financial support.

\section{References}

[1] A. Vasebi, M. Fesanghary, and S. M. T. Bathaee, "Combined heat and power economic dispatch by harmony search algorithm," International Journal of Electrical Power and Energy Systems, vol. 29, no. 10, pp. 713-719, 2007.

[2] M. Behnam, M. Mohammad, and R. Abbas, "Combined heat and power economic dispatch problem solution using particle swarm optimization with time varying acceleration coefficients," Electric Power Systems Research, vol. 95, pp. 9-18, 2013.

[3] P. Subbaraj, R. Rengaraj, and S. Salivahanan, "Enhancement of combined heat and power economic dispatch using self adaptive real-coded genetic algorithm," Applied Energy, vol. 86, no. 6, pp. 915-921, 2009.

[4] T. Guo, M. I. Henwood, and M. van Ooijen, "An algorithm for combined heat and power economic dispatch," IEEE Transactions on Power Systems, vol. 11, no. 4, pp. 1778-1784, 1996.

[5] A. Sashirekha, J. Pasupuleti, N. H. Moin, and C. S. Tan, "Combined heat and power (CHP) economic dispatch solved using Lagrangian relaxation with surrogate subgradient multiplier updates," Electrical Power and Energy Systems, vol. 44, pp. 421430, 2013.

[6] A. M. Jubril, A. O. Adediji, and O. A. Olaniyan, "Solving the combined heat and power dispatch problem: a semidefinite programming approach," Electric Power Components and Systems, vol. 40, pp. 1362-1376, 2012.

[7] V. N. Dieu and W. Ongsakul, "Augmented lagrangehopfield network for economic load dispatch with combined heat and power," Electric Power Components and Systems, vol. 37, no. 12, pp. 1289-1304, 2009.

[8] E. Khorram and M. Jaberipour, "Harmony search algorithm for solving combined heat and power economic dispatch problems," Energy Conversion and Management, vol. 52, no. 2, pp. 1550-1554, 2011.

[9] C. Su and C. Chiang, "An incorporated algorithm for combined heat and power economic dispatch," Electric Power Systems Research, vol. 69, no. 2-3, pp. 187-195, 2004.

[10] Y. H. Song, C. S. Chou, and T. J. Stonham, "Combined heat and power economic dispatch by improved ant colony search algorithm," Electric Power Systems Research, vol. 52, no. 2, pp. 115-121, 1999.

[11] S. S. Sadat Hosseini, A. Jafarnejad, A. H. Behrooz, and A. H. Gandomi, "Combined heat and power economic dispatch by mesh adaptive direct search algorithm," Expert Systems with Applications, vol. 38, no. 6, pp. 6556-6564, 2011.

[12] V. Ramesh, T. Jayabarathi, N. Shrivastava, and A. Baska, "A novel selective particle swarm optimization approach for combined heat and power economic dispatch," Electric Power Components and Systems, vol. 37, no. 11, pp. 1231-1240, 2009.
[13] M. Basu, "Artificial immune system for combined heat and power economic dispatch," Electrical Power and Energy Systems, vol. 43, pp. 1-5, 2012.

[14] M. Basu, "Bee colony optimization for combined heat and power economic dispatch," Expert Systems with Applications, vol. 38, no. 11, pp. 13527-13531, 2011.

[15] M. Basu, "Combined heat and power economic dispatch by using differential evolution," Electric Power Components and Systems, vol. 38, no. 8, pp. 996-1004, 2010.

[16] K. P. Wong and C. Algie, "Evolutionary programming approach for combined heat and power dispatch," Electric Power Systems Research, vol. 61, no. 3, pp. 227-232, 2002.

[17] N. Taher, A. A. Rasoul, R. Alireza, and A. Babak, "A new multi-objective reserve constrained combined heat and power dynamic economic emission dispatch," Energy, vol. 42, pp. 530$545,2012$.

[18] X. Xia and A. M. Elaiw, "Optimal dynamic economic dispatch of generation: a review," Electric Power Systems Research, vol. 80, no. 8, pp. 975-986, 2010.

[19] A. M. Elaiw, X. Xia, and A. M. Shehata, "Application of model predictive control to optimal dynamic dispatch of generation with emission limitations," Electric Power Systems Research, vol. 84, no. 1, pp. 31-44, 2012.

[20] G. P. Granelli, M. Montagna, G. L. Pasini, and P. Marannino, "Emission constrained dynamic dispatch," Electric Power Systems Research, vol. 24, no. 1, pp. 55-64, 1992.

[21] Y. H. Song and I. Yu, "Dynamic load dispatch with voltage security and environmental constraints," Electric Power Systems Research, vol. 43, no. 1, pp. 53-60, 1997.

[22] M. A. Abido, "Environmental/economic power dispatch using multiobjective evolutionary algorithms," IEEE Transactions on Power Systems, vol. 18, no. 4, pp. 1529-1537, 2003.

[23] M. Basu, "Dynamic economic emission dispatch using evolutionary programming and fuzzy satisfied method," International Journal of Emerging Electric Power Systems, vol. 8, pp. 1-15, 2007.

[24] M. Basu, "Dynamic economic emission dispatch using nondominated sorting genetic algorithm-II," International Journal of Electrical Power and Energy Systems, vol. 30, no. 2, pp. 140149, 2008.

[25] C. X. Guo, J. P. Zhan, and Q. H. Wu, "Dynamic economic emission dispatch based on group search optimizer with multiple producers," Electric Power Systems Research, vol. 86, pp. 8-16, 2012.

[26] A. M. Elaiw, X. Xia, and A. M. Shehata, "Hybrid DE-SQP and hybrid PSO-SQP methods for solving dynamic economic emission dispatch problem with valve-point effects," Electric Power Systems Research, vol. 84, pp. 192-200, 2013.

[27] A. M. Elaiw, X. Xia, and A. M. Shehata, "Minimization of fuel costs and gaseous emissions of electric power generation by model predictive control," Mathematical Problems in Engineering, vol. 2013, Article ID 906958, 15 pages, 2013.

[28] J. S. Alsumait, M. Qasem, J. K. Sykulski, and A. K. Al-Othman, "An improved Pattern Search based algorithm to solve the Dynamic Economic Dispatch problem with valve-point effect," Energy Conversion and Management, vol. 51, no. 10, pp. 20622067, 2010.

[29] M. Basu, "Particle swarm optimization based goal-attainment method for dynamic economic emission dispatch," Electric Power Components and Systems, vol. 34, no. 9, pp. 1015-1025, 2006. 
[30] P. Nicole, T. Anshul, T. Shashikala, and P. Manjaree, "An improved bacterial foraging algorithm for combined static/dynamic environmental economic dispatch," Applied Soft Computing, vol. 12, pp. 3500-3513, 2012.

[31] G. S. Piperagkas, A. G. Anastasiadis, and N. D. Hatziargyriou, "Stochastic PSO-based heat and power dispatch under environmental constraints incorporating CHP and wind power units," Electric Power Systems Research, vol. 81, no. 1, pp. 209-218, 2011.

[32] R. Storn and K. Price, "Differential Evolution-a simple and efficient adaptive scheme for global optimization over continuous spaces," Journal of Global Optimization, vol. 11, no. 4, pp. 341359, 1997.

[33] A. M. Elaiw, X. Xia, and A. M. Shehata, "Hybrid DE-SQP method for solving combined heat and power dynamic economic dispatch problem," Mathematical Problems in Engineering, vol. 2013, Article ID 982305, 7 pages, 2013.

[34] M. Basu, "Artificial immune system for dynamic economic dispatch," International Journal of Electrical Power and Energy Systems, vol. 33, no. 1, pp. 131-136, 2011.

[35] X. Xia, J. Zhang, and A. Elaiw, "An application of model predictive control to the dynamic economic dispatch of power generation," Control Engineering Practice, vol. 19, no. 6, pp. 638648, 2011.

[36] C. K. Panigrahi, P. K. Chattopadhyay, R. N. Chakrabarti, and M. Basu, "Simulated annealing technique for dynamic economic dispatch," Electric Power Components and Systems, vol. 34, no. 5, pp. 577-586, 2006.

[37] P. Attaviriyanupap, H. Kita, E. Tanaka, and J. Hasegawa, "A hybrid EP and SQP for dynamic economic dispatch with nonsmooth fuel cost function," IEEE Transactions on Power Systems, vol. 17, no. 2, pp. 411-416, 2002.

[38] P. T. Boggs and J. W. Tolle, "Sequential quadratic programming," Acta Numerica, vol. 3, no. 4, pp. 1-52, 1995. 


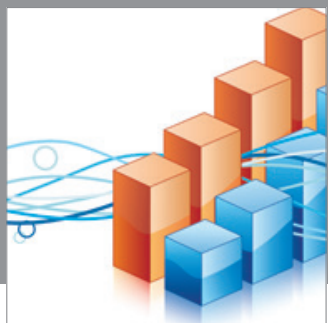

Advances in

Operations Research

mansans

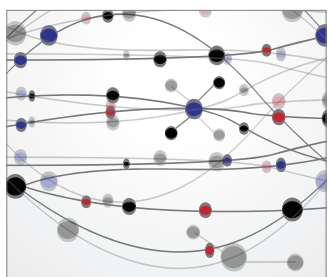

The Scientific World Journal
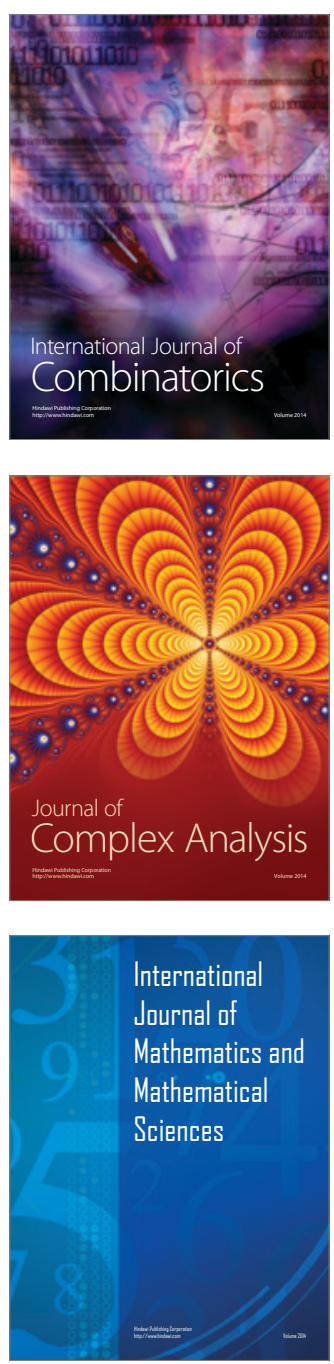
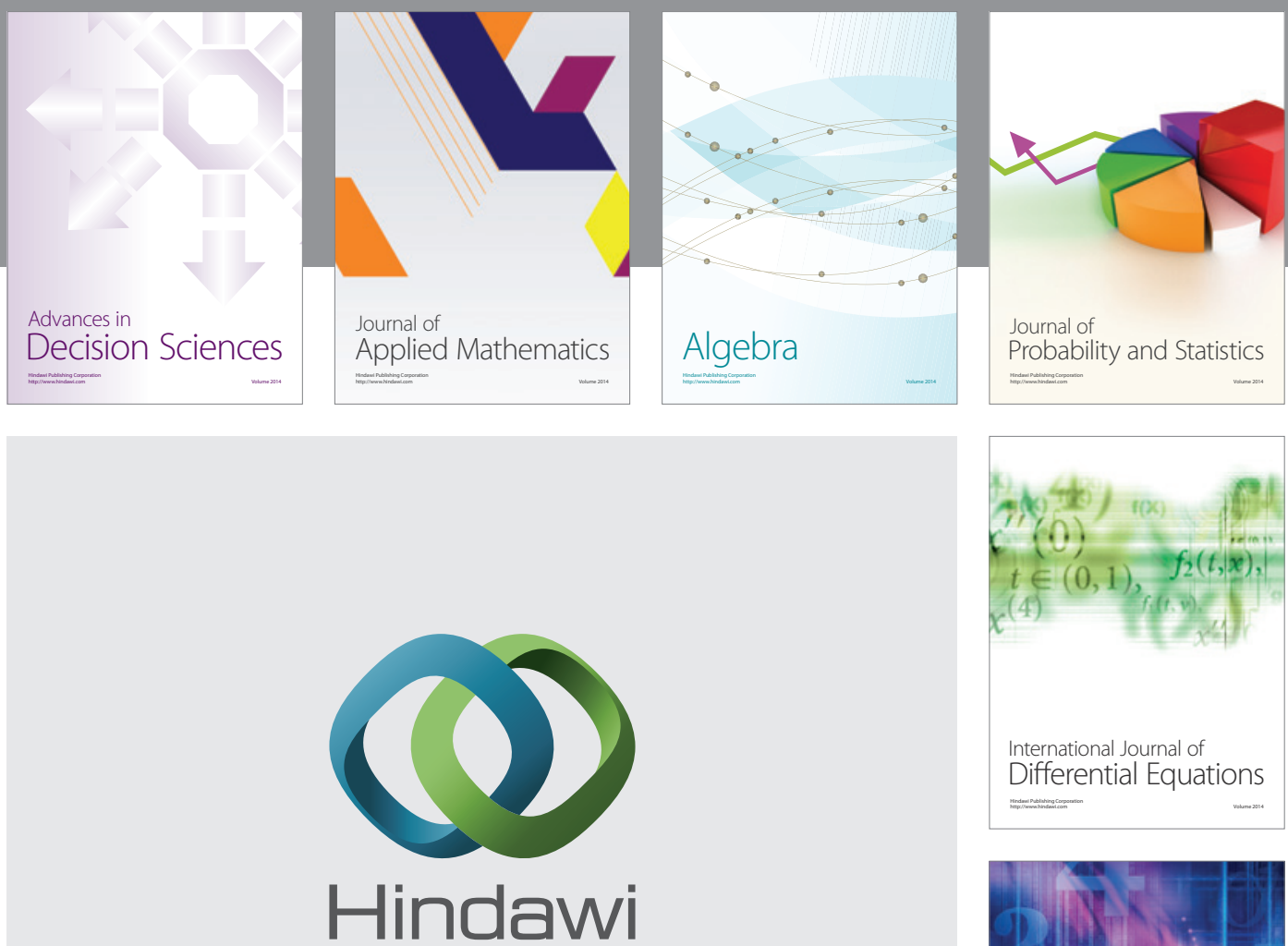

Submit your manuscripts at http://www.hindawi.com
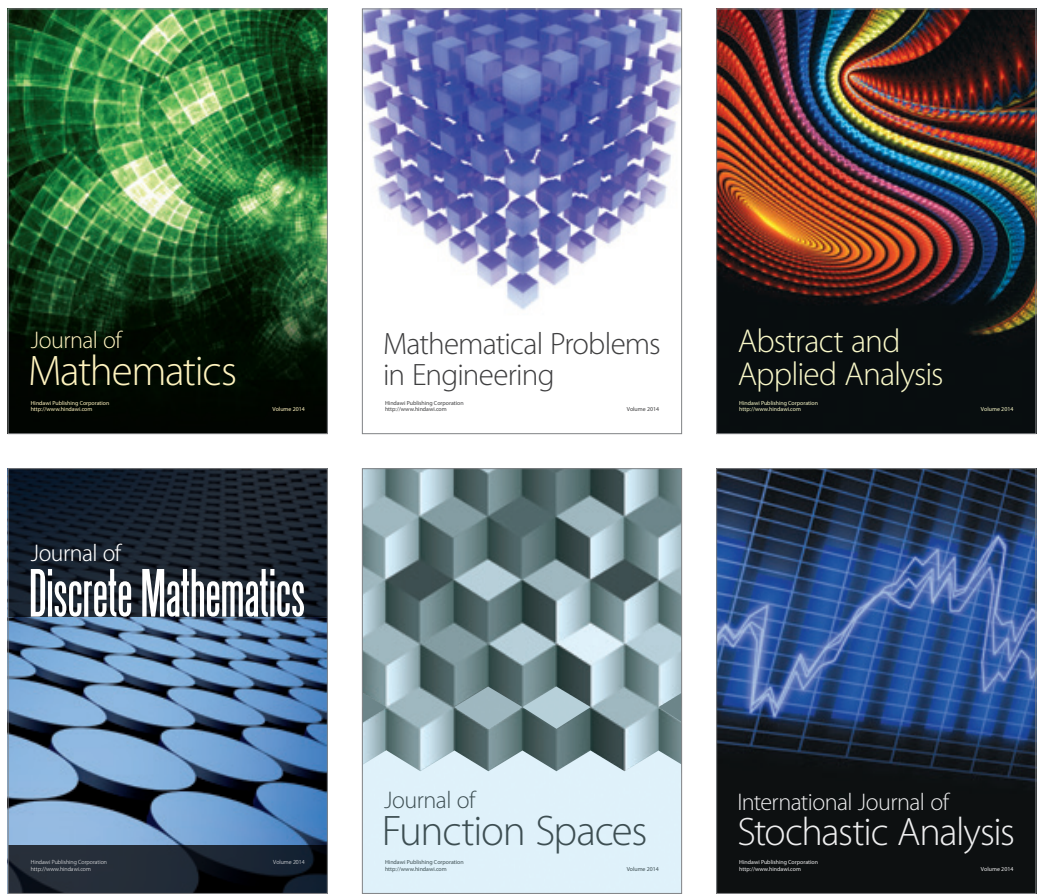

Journal of

Function Spaces

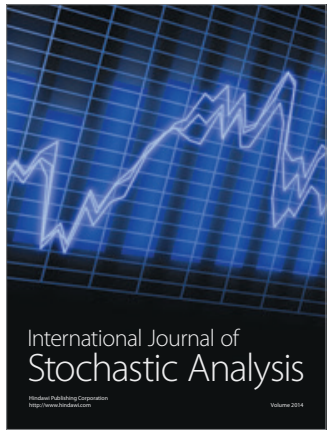

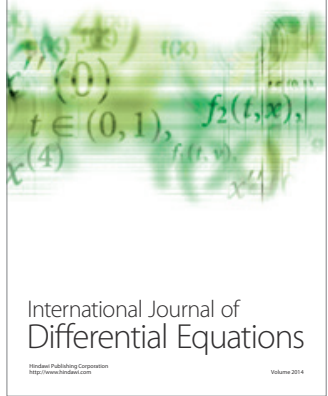
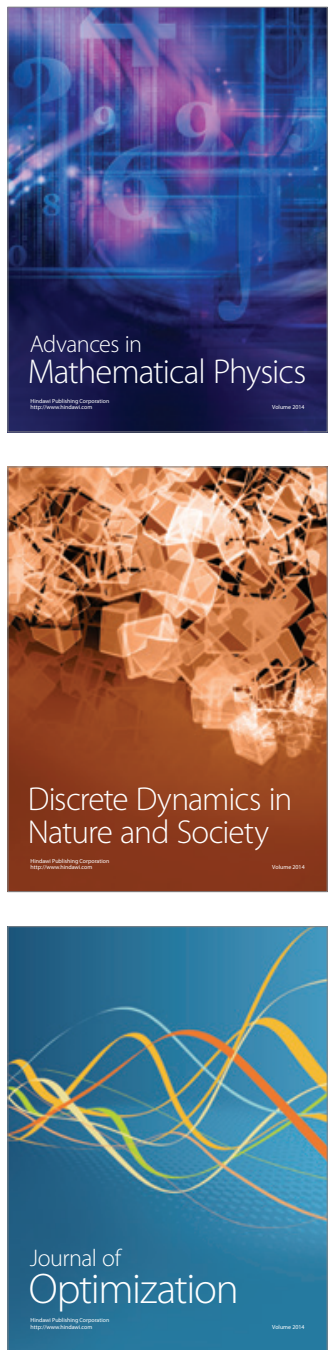